\title{
Research Hotspots Analysis of Electronic Health
}

\author{
Wen-Tao WANG ${ }^{1, a}$, Kun ZHANG ${ }^{1, b,{ }^{*}}$, Jing LI $^{1}$, Yun-Zhi WANG ${ }^{2}$, Shuai ZHANG ${ }^{1}$, \\ Xiao $\mathrm{XIE}^{1}$ and Yang-Qun $\mathrm{XIE}^{3}$ \\ ${ }^{1}$ School of Management, Anhui University, Hefei 230601, China \\ ${ }^{2}$ School of Information Management, Wuhan University, Wuhan 430072, China \\ ${ }^{3}$ Hefei Normal University, Hefei 230601, China \\ awwtor007@163.com, bzhangkun_wys@163.com \\ ${ }^{*}$ Corresponding author
}

\begin{abstract}
Keywords: Electronic health (eHealth), Electronic health record (EHR), Mobile health (mHealth), Electronic health service, Electronic health literacy.
\end{abstract}

\begin{abstract}
Purpose/significance] Aims to summarize the current hotspots of international electronic health research and provide some reference for the future development of electronic health. [Method/process] By using the methods of co-word analysis, cluster analysis and content analysis, to summarize the research hotspots in the field of electronic health. [Result/Conclusion] Most of the current researches focus on seven aspects, they are the importance of electronic health, electronic health records, information technology applied to electronic health, obstacles to the development of electronic health, mobile health, electronic health services and electronic health literacy.
\end{abstract}

\section{Introduction}

Health is an inevitable requirement for the comprehensive development and the ultimate aim of mankind. However, the World Health Organization (WHO) survey shows that there are only about $5 \%$ people who are in a ideal health condition, sub-health and various chronic diseases have become major threats to human health. With the increase of social population, the proportion of sub-healthy people is still rising. In addition, with the modern information technology applied to the medical field and the improvement of people's health awareness, people start to have higher demands on the quality of health. In this context, electronic health emerges.

At present, there is no uniform definition on electronic health(eHealth). Some scholars believe that eHealth refers to the combination of electronic communication and information technology in the field of healthcare, whether in short or long distance, it can achieve the electronic transmission, storage and retrieval of data, and can be used for clinical, education and management[1] Some scholars insist that eHealth is a service that uses information technology to provide more quality and efficiency for healthcare[2]. And Some scholars believe that eHealth is a new type of business model which is formed by the expansion of electronic commerce using internet technology in healthcare and pharmaceuticals[3]. It can be seen that a thousand people see a thousand Hamlet. Combined with the definition of predecessors, the author argues that eHealth is a collective term for a series of service processes which uses information and communication technology tools, to help and strengthen the prevention, diagnosis, monitoring, treatment and management in the field of healthcare.

As the importance of eHealth in promoting the modernization of health system, it has become an important way to alleviate the doctor-patient relationship and crate a new trend of the development of healthcare system. Based on the current situation, the antuor systematically analyzes the international eHealth related paperes by using the methods of co-word analysis, cluster analysis and content analysis. Through the analysis of international eHealth research hotspots, the aim of this paper is to provide a reference for the future development of eHealth. The research process of the paper is shown in Figure 1. 


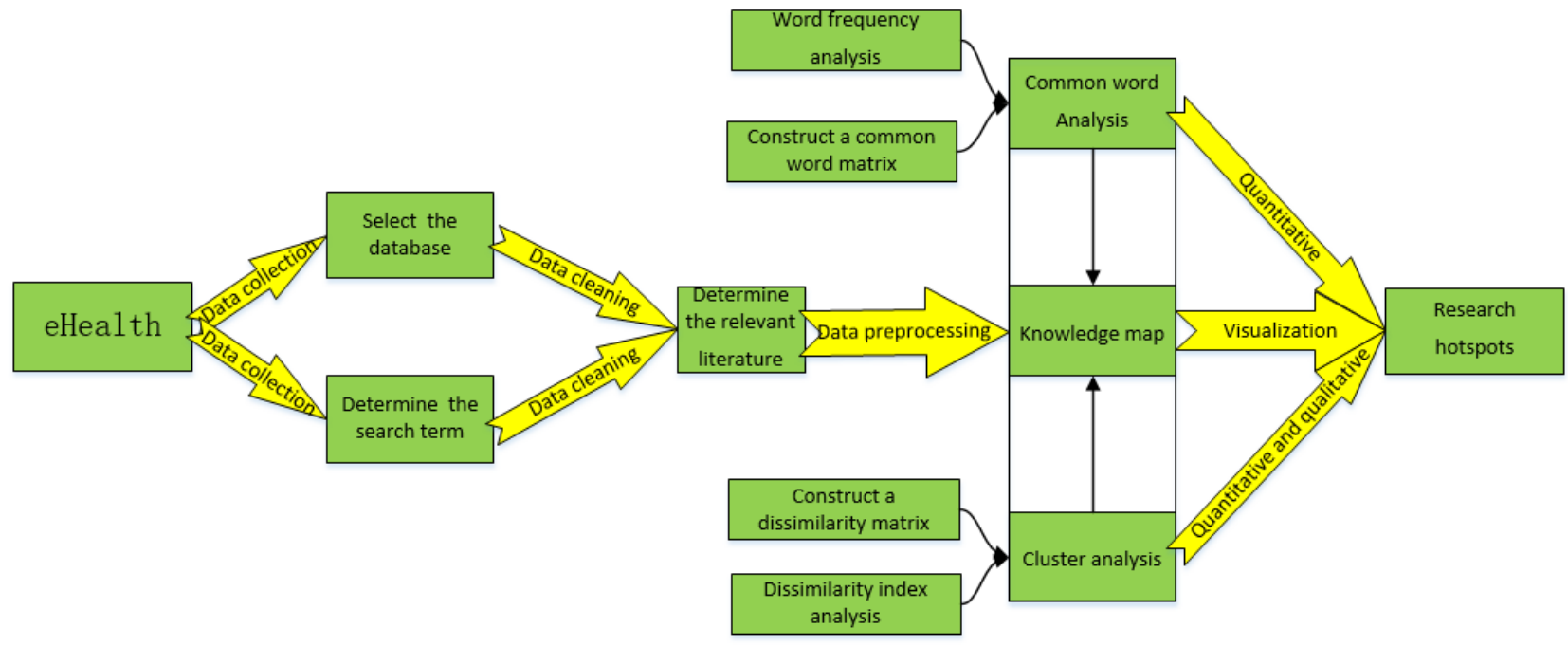

Fig. 1 Research process

\section{Data Sources and Research Overview}

The author takes the web of Science core collection database as the data acquisition platform, with "electronic health" or "eHealth" or "E-health" as the search term, the periodical source is set to "SSCI", the literature type is set to "Article", then refining 4057 articles. These articles are also the analysis samples of this paper. After statistical analysis of the time distribution of these documents, Figure 2 is obtained. It can be seen researchers pay much more attention on the study of electronic health in recent years. Based on the linear prediction of current research trends, the author believes that the volume of future related research will keep sustaiable growth, and the related topics of eHealth should be an important field for researchers to pay attention to.

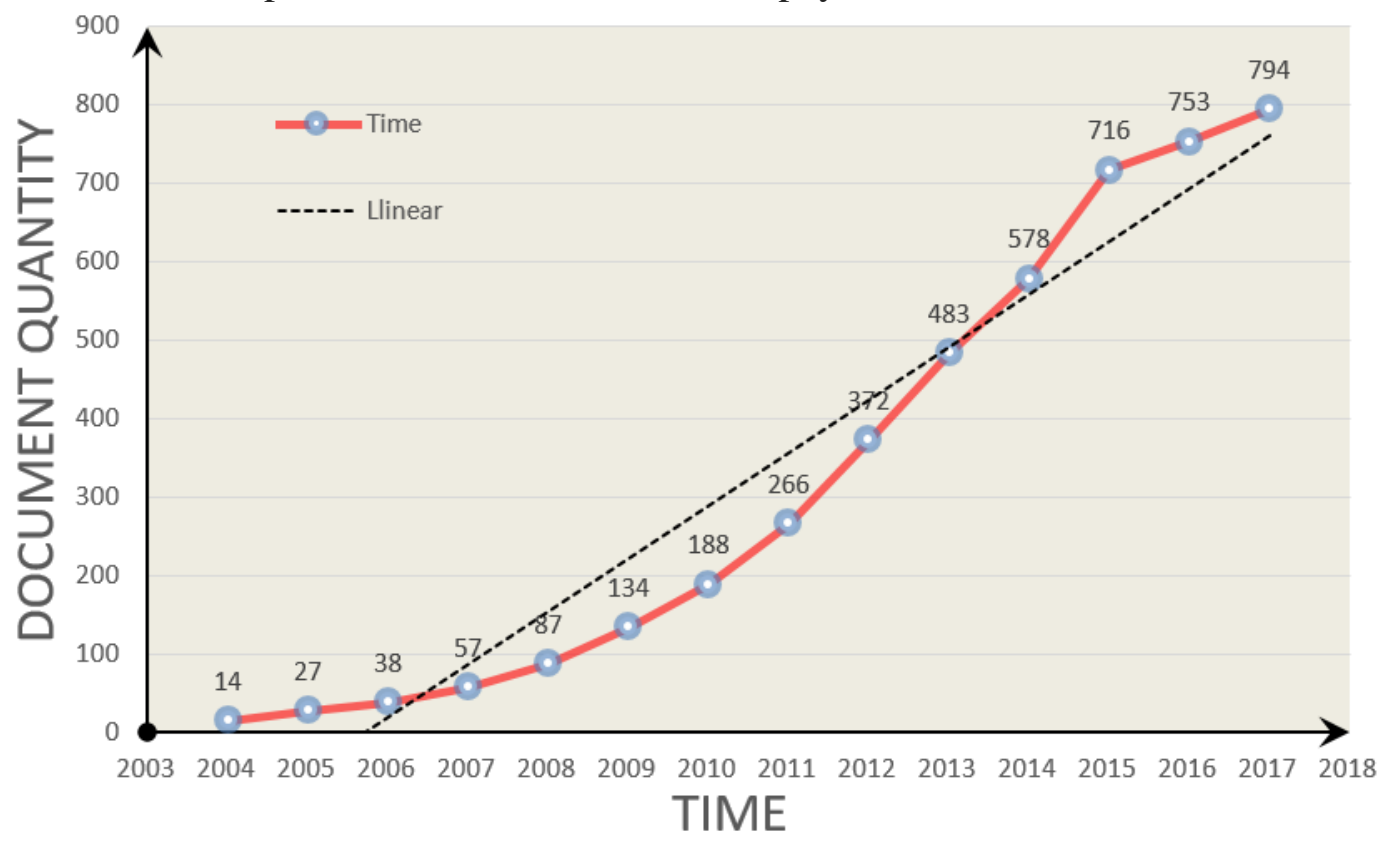

Fig. 2 Annual distribution of eHealth literature

\section{Research Hotspots Analysis}

Through the co-word analysis, cluster analysis and content analysis method, the related literatures are summarized and seven topics are extracted, as shown in the figure 3. 


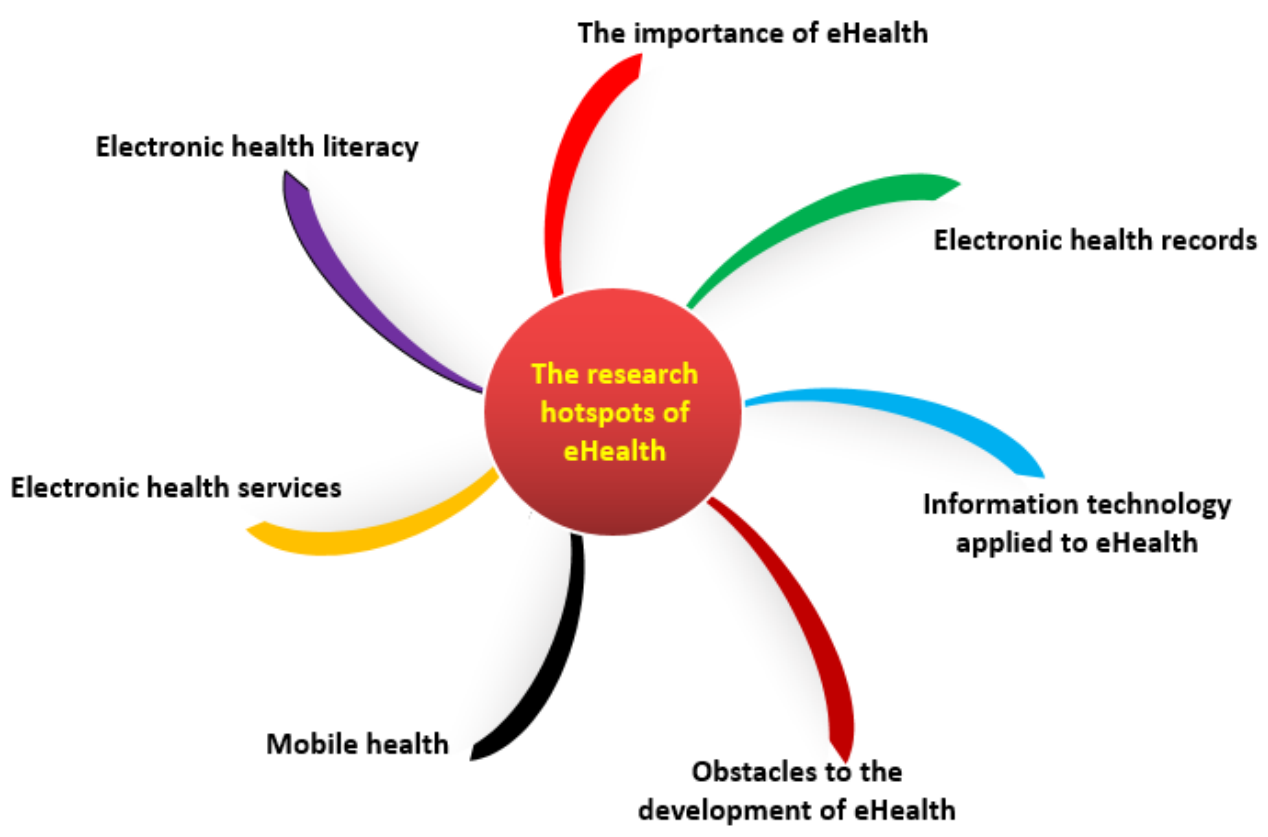

Fig. 3 EHealth research hotspots

\section{The Importance of eHealth}

The development of eHealth is an important way to improve the level of human health, which is of great significance to the development of human society.

First of all, it helps to reduce information asymmetry and to narrow the information gap between doctors and patients. The sharing of health knowledge among different users can help to increase the patient's health knowledge, and help doctors and patients have reduce the conflicts between doctors and patients.

Secondly, it helps to reduce the medical expense and improve the efficiency of medical service. Through information sharing and interoperability to avoid duplication of user information, eHealth not only helps to reduce the cost of patients, but also helps to improve the service efficiency of medical institutions.

Finally, it helps to meet the personalized health needs of users. EHealth enables users to reduce the limitation of time and space on doctors' treatment. Users can not only receive medical health information or services which provided by global health providers whenever and wherever, but also can achieve customized health management requirements and more personalized health demands.

\section{Electronic Health Records (EHR)}

The Electronic Health Record(EHR), also known as Electronic Medical Record (EMR), it refers to the use of computers to create, process and save information on personal health status and health care records[4]. Based on medical information resources, EHR is aiming at preventing excessive medical treatment, reducing and ultimately eliminating medical errors, reducing medical costs and improving the level of medical care. It helps to realize the interactive systems and interactive platforms sharing of medical information, and provides important medical data for patients ' timely and effective treatment.

\section{Information Technology Applied to eHealth}

Information technology aims to improve the health status of users and even heal life-saving medical targets by coordinating the relationship between doctors, patients, medical equipment suppliers and other participants. With the integration of Internet, big data, cloud computing and other technologies, many medical problems are solved.

Firstly, the application of IoT technology in the field of eHealth makes medical informatization a qualitative flyover, which contributes to realize the continuous, flexible, individual and 
trans-regional telemedicine health services. Based on the IoT technology, a series of medical related products such as medical sensors and wearable devices continue to emerge. Through the use of these medical products, health services in the scope of medical institutions have been effectively expanded. It also make the user's health status be real time monitoring and intelligent assessment, thereby increasing the safety factor of users[5].

Secondly, big data technology has a great effect on improving medical value[6]. The use of big data technology to integrate and analyze the user's personal EHR and the family EHR information enables the whole life cycle management of the user's personal health to be managed at any time and any place, so as to ensure the continuity and consistency of the user's health information.

Thirdly, the use of cloud computing technology can realize the user health information circulation sharing and help to improve the service efficiency and service quality of medical institutions[7]. It also helps to protect the users' information security and prevent leakage of personal privacy of users[8].

\section{Obstacles to the Development of eHealth}

As a multidisciplinary area, eHealth faces many obstacles to its development.

First of all, the lack of uniform standards leads to difficulties in collaborating with the parties[9]. Because of the lack of unified policy standards, technical standards and data standards, the relevant departments in the electronic health application process fragmented, it is difficult to achieve information sharing and interoperability.

Secondly, information security and user privacy protection barriers. In the electronic health environment, the users' personal health information is shared among the participating parties, and it is possible to disclose the data in any one link, which undoubtedly increases the possibility of information insecurity.

Finally, the digital divide. The in-depth development of eHealth has virtually widened the digital divide between digital natives[10], digital immigrants[11] and digital refugees[12] users, making the digital divide more difficult to bridge.

\section{Mobile Health(mHealth)}

In accordance with the definition given by the Healthcare Information and Management Systems Society (HIMSS), mobile health[13](mHealth)refers to a healthcare model that provides medical services and information through the use of mobile communications technologies such as mobile phones, satellite communications and PDAs. The real value of mHealth is to overcome the limitation of time and space on doctors' diagnosis and provide continuous, flexible and efficient medical services, which opens up a wide space for the development of modern medicine.

According to the survey, chronic disease control has become the biggest challenge in the global medical system[14]. Since chronic disease management requires patients to keep in touch with the hospital for a long time, and one of the important efficacy of mHealth is to realize real time monitoring of the patients ' condition. Therefore, the advent of mHealth brings new opportunities for users of chronic disease.

In addition, the application of mHealth can help to alleviate the contradiction between the imbalance of medical resources and the increasingly strong health needs of users, and provide users with convenient access to medical treatment. With the application of wearable devices in the medical field, the number of users and the stickiness of users in mHealth mode will increase dramatically and mHealth is expected to usher in a new round of tuyere.

\section{EHealth Services}

In recent years, eHealth services are moving in the direction of personal health and grassroots participation. They are close to the service audience groups themselves and attach importance to the feedback and interaction of health information. The development of medical service mode has changed from "3P" mode to the "4P" [15] mode, which is to pay more attention to public and social participation. That is, on the basis of prevention, prediction and individuality, it increases the user's participation. 
The focus of medical health services in various countries has also gradually shifted from disease control and management to the prevention users' health and disease. Through the analysis of users 'big data of eHealth platform and resource sharing of relevant eHealth information system, the medical institutions can provide patients with scientific decisions and suggestions while respecting patients' wishes and choices. So the medical health services mode is oriented towards a more humane service direction.

\section{EHealth Literacy}

Electronic health literacy refers to the ability of users to search, find, evaluate, integrate and apply the information needed to solve health problems in the Internet environment[16].

In the process of eHealth services, the electronic health literacy of the two main beneficiary groups which are doctors and patients, plays an important role in the development of eHealth. The willingness and ability of these two subjects to participate in eHealth have greatly influenced and even determined the level of eHealth service.

With the rapid development of the new generation of information technology, the health information received by people has the characteristics of being dynamic and changeable. Therefore, the eHealth literacy is necessary for everyone in the era of great health and the importance of cultivating users' eHealth literacy is self-evident.

\section{Conclusions}

The paper systematically summarizes the international eHealth related researches, and draws the conclusion that the current international eHealth researches mainly focus on seven aspects, they are the importance of eHealth, eHealth records, information technology applied to eHealth, obstacles to the development of eHealth, mHealth, eHealth services and eHealth literacy.

Overall, the contents of international eHealth research are rich, comprehensive, extensive and developmental, but there are also some deficiencies. Firstly, the analysis of the status of precision medical treatment is less. Secondly, there is a lack of relevant research on virtual communities that are closely linked to eHealth. Thirdly, less attention has been paid to the application of new information technology in the field of eHealth. Finally, the researchers' study on the eHealth is enthusiastic, but the homogeneity of research topics is prominent.

As a product of cross-border integration of information technology and healthcare, eHealth has become an inevitable trend in the future development of the medical field. So it is necessary to conduct an in-depth exploration on eHealth related topics. It is expected that through the systematic introduction of the paper, the authors can provide ideas for the future development of eHealth.

\section{Acknowledgements}

This research has been supported by grants from the National Natural Science Foundation of China (71273109) and the Philosophy and Social Sciences Planning Project of Anhui Province(AHSKY2017D16).

\section{References}

[1] Mitchell J. From telehealth to e-health: The unstoppable rise of e-health. Canberra, Australia: Commonwealth Department of Communications[J]. Information Technology and the Arts (DOCITA), 1999.

[2] Eysenbach G. What is e-health? [J]. Journal of medical Internet research, 2001, 3(2):20-21.

[3] Loman P. E-Health: Putting health on the Net[J]. An FCG White Paper. URL: http://www. fcg. com/webfiles/whitepaper/white_paper_files/wpEhealth. Asp[accessed 2004 June 24], 1999.

[4] Strobel C D. American recovery and reinvestment act of 2009[J]. Journal of Corporate Accounting \& Finance, 2009, 20(5): 83-85. 
[5] Al-Majeed S S, Al-Mejibli I S, Karam J. Home telehealth by internet of things (IoT)[C]//Electrical and computer engineering (CCECE), 2015 IEEE 28th Canadian conference on. IEEE, 2015: 609-613.

[6] Chen H, Chiang R H L, Storey V C. Business intelligence and analytics: From big data to big impact[J]. MIS quarterly, 2012, 36(4) ):1165-1188.

[7] Devaraj S, Ow T T, Kohli R. Examining the impact of information technology and patient flow on healthcare performance: A Theory of Swift and Even Flow (TSEF) perspective[J]. Journal of Operations Management, 2013, 31(4): 181-192.

[8] Klein C A. Cloudy confidentiality: clinical and legal implications of cloud computing in health care[J]. Journal of the American Academy of Psychiatry and the Law Online, 2011, 39(4): 571-578.

[9] Pagani M. Digital business strategy and value creation: Framing the dynamic cycle of control points[J]. Mis Quarterly, 2013, 37(2):617-632.

[10]Palfrey J G, Gasser U. Born digital: Understanding the first generation of digital natives[M]. Basic Books, 2013.

[11]Prensky M. Digital natives, digital immigrants part 1[J]. On the horizon, 2001, 9(5): 1-6.

[12]Combes B. Generation Y: Are they reaslly digital natives or more like digital refugees?[J]. School Library Assoc of Victoria, 2008.

[13]Källander K, Tibenderana J K, Akpogheneta O J, et al. Mobile health (mHealth) approaches and lessons for increased performance and retention of community health workers in low-and middle-income countries: a review[J]. Journal of medical Internet research, 2013, 15(1):e17.

[14]Jara A J, Moreno-Sanchez P, Skarmeta A F, et al. IPv6 addressing proxy: Mapping native addressing from legacy technologies and devices to the Internet of Things (IPv6)[J]. Sensors, 2013, 13(5): 6687-6712.

[15]Hood L, Flores M. A personal view on systems medicine and the emergence of proactive P4 medicine: predictive, preventive, personalized and participatory[J]. New biotechnology, 2012, 29(6): 613-624.

[16]Norman C D, Skinner H A. eHealth literacy: essential skills for consumer health in a networked world[J]. Journal of medical Internet research, 2006, 8(2): e9. 